\title{
CLINICAL RESEARCH ARTICLE Surfactant and budesonide for respiratory distress syndrome: an observational study
}

\author{
T. Brett Kothe ${ }^{1,2}$, Farouk H. Sadiq ${ }^{1}$, Nikki Burleyson ${ }^{3}$, Howard L. Williams ${ }^{3}$, Connie Anderson ${ }^{1}$ and Noah H. Hillman ${ }^{1}$
}

BACKGROUND: In preterm infants on moderately high ventilator support, the addition of budesonide to surfactant lowered bronchopulmonary dysplasia (BPD) rates by $20 \%$ without increased morbidity or mortality. The aim of this cohort comparison was to determine the safety and efficacy of the combination in infants with milder respiratory distress syndrome (RDS).

METHODS: In August 2016 we began administering budesonide $(0.25 \mathrm{mg} / \mathrm{kg}$ ) mixed with surfactant (Survanta $4 \mathrm{~mL} / \mathrm{kg})$ to all infants $\leq 1250 \mathrm{~g}$ who failed CPAP and required intubation. Infants were compared to a historical cohort (2013-2016) who received surfactant alone.

RESULTS: BPD or death did not change between the historical surfactant cohort $(71 \%, n=294)$ and the budesonide cohort $(69 \%$, $n=173)$. Budesonide was associated with a decrease in the need for continued mechanical ventilation, severe BPD type II or death $(19-12 \%)$, grade III BPD or death $(31-21 \%)$, and the median gestational age at discharge was 1 week earlier. Histologic chorioamnionitis was associated with decreased budesonide effects. Secondary morbidities (NEC, IVH, ROP, Sepsis) were similar. CONCLUSION: Overall BPD rates remained unchanged with the addition of budesonide. Budesonide was associated with decreased severity of BPD, decreased mechanical ventilation use, earlier discharge, and similar short-term outcomes.

Pediatric Research (2020) 87:940-945; https://doi.org/10.1038/s41390-019-0663-6

\section{INTRODUCTION}

Lung inflammation is central to the pathophysiology of bronchopulmonary dysplasia (BPD) and anti-inflammatory medications, such as systemic corticosteroids, can decrease BPD. ${ }^{1-4}$ However, there is a risk of long-term adverse outcomes with systemic steroid use. ${ }^{4}$ Delivery of steroids directly to the lungs, with either inhaled budesonide or budesonide instilled with surfactant, have recently been shown to decrease BPD. ${ }^{5-7}$ Budesonide is a corticosteroid commonly used to treat lung inflammation in asthma and is believed to remain primarily in the airways as budesonide esters. ${ }^{8,9}$ Inhaled budesonide has been shown to decrease the risk of BPD when started early in life, ${ }^{10,11}$ but it was also shown to increase mortality. ${ }^{5}$ However, when budesonide was mixed with a treatment dose of surfactant, it reduced the rate of BPD by $20 \%$ without increased mortality or adverse physical or neurologic outcomes. ${ }^{7,12,13}$ To receive budesonide mixed with surfactant in these trials, infants required significant respiratory support on mechanical ventilation and a fraction of inspired oxygen $\left(\mathrm{FiO}_{2}\right)$ requirement of $>0.5 .^{7,12,13}$ Since BPD can occur in infants with milder lung disease, understanding the role of budesonide with surfactant in all infants at risk for BPD is critical.

Aerosolized budesonide remains primarily in the proximal airways, whereas surfactant transports the budesonide throughout the distal lung, where the alveolar simplification of BPD occurs. ${ }^{6,7,14}$ The combination of budesonide and surfactant improves gas exchange, matures the lung, and reduces lung inflammation in respiratory distress syndrome (RDS) models. ${ }^{6,7,14-17}$ The half-life of budesonide in the fetal lung is about $4 \mathrm{~h}$, and systemically released budesonide should be rapidly metabolized in the liver. ${ }^{12,18-20}$
Budesonide is detected in the plasma of infants and preterm sheep given budesonide mixed with surfactant. $7,16,17,21$

With a desire to decrease the rate of BPD in our NICU, we made a local practice change to administer the combination of surfactant $(4 \mathrm{~mL} / \mathrm{kg})$ and budesonide $(0.25 \mathrm{mg} / \mathrm{kg})$ to infants $\leq$ $1250 \mathrm{~g}$ who failed noninvasive respiratory support for respiratory distress or were intubated for resuscitation. ${ }^{7,12,13}$ Some limitations on the generalizability of the studies by Yeh et al. were the severity of the lung disease prior to treatment and the racial distribution within the studies, which were primarily conducted in Taiwan. ${ }^{7,12}$ We now present a large, 2-year cohort of infants, with an ethnic distribution resembling many NICUs in the United States, who failed noninvasive ventilation and received budesonide mixed with surfactant. In instituting the local practice change to include budesonide with surfactant, our objective was to decrease our moderately high BPD rate, while confirming the safety of the combination demonstrated in the Yeh studies. The primary outcomes of BPD or death and severity of BPD, and secondary neonatal morbidity outcomes, were compared with a 3year historical cohort of patients who received only surfactant.

\section{METHODS}

The Division of Neonatology made a local clinical practice change in 2016 to include budesonide with surfactant. The ethics committee at Cardinal Glennon Children's Hospital was also consulted about need for consent for the local change in clinical care. Initial data were collected prospectively as a quality improvement initiative to assess the clinical practice change.

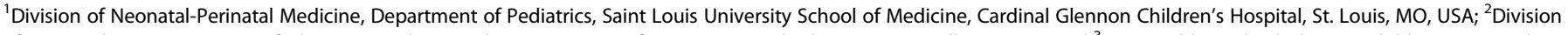

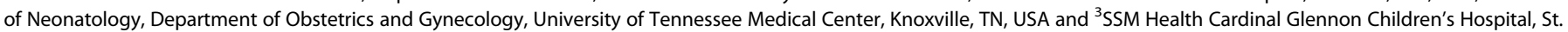
Louis, MO, USA

Correspondence: Noah H. Hillman (Noah.Hillman@health.slu.edu)

Received: 13 March 2019 Revised: 3 September 2019 Accepted: 5 October 2019

Published online: 12 November 2019 
Retrospective data collection and analysis on the infants after discharge, from both the surfactant alone and budesonide mixed with surfactant groups, was utilized to determine efficacy after approval by the Saint Louis University Institutional Review Board (IRB\# 28729).

Beginning on August 1, 2016, our level III/IV NICUs at SSM Health Cardinal Glennon Children's Hospital and St. Mary's Hospital began to administer budesonide $0.25 \mathrm{mg} / \mathrm{kg}$ (Pulmicort Respules $0.5 \mathrm{mg} / 2 \mathrm{~mL}$, AstraZeneca Pharmaceuticals) mixed with beractant surfactant $4 \mathrm{~mL} / \mathrm{kg}$ (Survanta, $100 \mathrm{mg} / \mathrm{kg}$, AbbVie) to all freshly born infants $\leq 1250 \mathrm{~g}$ who were intubated and would have normally received surfactant alone. Our clinical practice, regardless of gestational age (GA) or weight, is an initial trial of continuous positive airway pressure (CPAP) with bubble CPAP using a RAM cannula interface and noninvasive ventilation failure guidelines similar to the SUPPORT trial are used for infants less than 32 weeks $\mathrm{GA}^{22,23}$ Very preterm infants who fail CPAP within the first $24 \mathrm{~h}$ of life or are intubated for resuscitation typically receive surfactant. To prepare the combination, surfactant and budesonide were drawn up into separate syringes. The budesonide was pushed into the surfactant syringe through a plastic connector and the combination was then gently rotated a few times. The addition of budesonide to surfactant at these ratios does not affect the surface tension reduction properties ${ }^{7,12}$ and was administered similar to surfactant alone. As in the Yeh et al. study, infants could receive additional doses of budesonide with surfactant every $8 \mathrm{~h}$ after the first dose if the $\mathrm{FiO}_{2}$ remained $\geq 0.30$ and the infant required high pressures to maintain tidal volume ventilation. ${ }^{7}$ Although rarely given, surfactant doses after the first few days would not include budesonide.

Infants who received the combination of budesonide and surfactant (August 2016 to August 2018) were compared to an historical cohort of infants born $\leq 1250 \mathrm{~g}$ who received surfactant alone (August of 2013 until July of 2016). Infants born <23 weeks gestation, $<500 \mathrm{~g}$, who died within first $12 \mathrm{~h}$, or with congenital anomalies were excluded. Data were extracted from EPIC using Microsoft SQL server and verified via chart review. Placental pathology was reviewed where available (88\% of infants) and chorioamnionitis was defined histologically as placental inflammation or funisitis. Antenatal corticosteroids (ANCS) were defined as given if even a partial course was completed. Outborn was defined as not being born at our delivery hospital (St. Mary's). The clinical risk index for babies (CRIB) II scores were calculated. ${ }^{24}$

Our primary outcomes were the incidence of moderate to severe BPD or death and the severity of BPD. BPD was defined as the need for oxygen on day of life (DOL) 28 and any level of respiratory support at 36 weeks corrected gestational age (CGA). ${ }^{25,26}$ BPD was then further subcategorized by severity: (1) No BPD: room air or CPAP with an $\mathrm{FiO}_{2}$ of 0.21 at DOL 28 and no respiratory support at 36 weeks CGA; (2) Mild: respiratory support at DOL 28 and room air at 36 weeks CGA; (3) Moderate BPD: $\mathrm{FiO}_{2}<0.30$ at 36 weeks CGA; (4) Severe Type $1: \mathrm{FiO}_{2} \geq 0.30$ or the need for CPAP at 36 weeks CGA; (5) Severe Type 2: noninvasive positive pressure ventilation (NIPPV) or mechanical ventilation at 36 weeks CGA. ${ }^{27}$ In our unit, infants around 35 weeks CGA who require nasal cannula are often changed to an $\mathrm{FiO}_{2}$ of 1.0 with air flows weaned prior to discharge. BPD severity was further categorized based on severity grades as outlined by NICHD Workshop in $2018 .^{28}$ Secondary respiratory outcomes measured included necessity for respiratory support and $\mathrm{FiO}_{2}$ requirements (DOL 3, DOL 7, DOL 28, and discharge), BPD risk at DOL 7 based on the NICHD BPD Outcome Estimator (neonatal.rti.org/index.cfm), ${ }^{1}$ and the need for high-frequency oscillatory ventilation (HFOV).

Nonrespiratory secondary outcomes measured included death rate, length of stay (LOS), and growth parameters (daily weights and length on infant stadiometer). Our institution initiated a feeding protocol in 2012 with early enteral feeds with mother or donor breast milk, trophic feeds for 3 days, then daily advancement of feeds to $160 \mathrm{~mL} / \mathrm{kg} /$ day of $24 \mathrm{kcal} /$ ounce milk until 32 weeks CGA. Our unit's written protocol recommends a 9day dexamethasone taper for infants with a risk of moderate or severe BPD or death that is $>60 \%$ at DOL 7 based on the NICHD calculator. $^{29}$ Dexamethasone use was defined as greater than three doses. Necrotizing enterocolitis (Bell stage 2 or greater), ${ }^{30}$ spontaneous intestinal perforation, pneumothoraces, pulmonary interstitial emphysema, intraventricular hemorrhage (IVH), and periventricular leukomalacia (PVL) were determined from radiographic reports. All echocardiogram reports were reviewed for any patent ductus arteriosus (PDA). Echocardiograms and treatment of a PDA (indomethacin, ibuprofen, or ligation) were directed by the medical team. Retinopathy of prematurity (ROP) stage and treatment (laser therapy or bevacizumab) was extracted from Ophthalmology notes. ${ }^{31}$ Gastrostomy tube placement, tracheostomy, neurosurgical intervention, pulmonary hemorrhage, cultureproven sepsis during hospitalization, hyperglycemia requiring insulin administration, and the use of sildenafil for pulmonary hypertension were extracted from medical record. Length is measured weekly with a length board (stadiometer) and dietitians round with the clinical teams. Risk factors predictive of poor neurologic outcomes: (1) BPD, (2) ROP stage 4, 5 or treatment, and (3) IVH grade 3 or 4 or PVL were compared between cohorts. ${ }^{32,33}$

\section{Statistical analysis}

Numerical data were analyzed with Student's $t$ tests or MannWhitney tests when values were not uniformly distributed. Results are displayed as mean \pm standard deviation or median (25th quartile, 75th quartile). Dichotomous data were analyzed by chi-squared test with a two-tailed $p$ value $<0.05$ considered significant. Multivariable logistic regression was performed and was adjusted for gestational age, birth weight, gender, race, Apgar score, antenatal steroids, histologic chorioamnionitis, and surfactant dosing. Clinical or significant variables were forced into the linear regression model (Supplementary Table 1). The secondary analysis based on chorioamnionitis and birth weight was planned based on their associations with BPD. Statistics were generated on GraphPad Prism 6, Vassarstats.net, and IBM SPSS.

\section{RESULTS}

There were 294 infants in the historical surfactant cohort and 173 infants in the surfactant with budesonide cohort (Table 1). There were no differences in gestational age, birth weight, gender, race, outborn status, antenatal steroid usage, histologic chorioamnionitis, Agpar scores, CRIB II scores, or doses of surfactant between the two cohorts.

Primary outcome (BPD or death)

Infants in the budesonide cohort compared to the surfactant cohort had a similar rate of moderate to severe BPD or death (odds ratio (OR) 0.94; 95\% confidence interval [Cl], 0.62-1.42; $p=$ 0.75 ) (Table 2). There was no difference in the percentage of patients that died. Severe BPD Type 2 or death was lower in the budesonide cohort compared to surfactant cohort (OR 0.54; $0.31-0.94 ; p=0.03$ ), as was evident by a decreased need for mechanical ventilation or NIPPV at 36 weeks CGA (Table 2). More infants in the budesonide cohort were also on either nasal cannula or room air at 36 weeks (OR 1.6; 1.03-2.4; $p=0.04$ ). An NIH workshop on BPD recommended in 2018 a revised grading scale for severity of BPD. ${ }^{28}$ Using this new severity scoring, Grade 3 BPD or death was decreased in the budesonide cohort (OR 0.5; $0.38-0.91 ; p=0.02$ ), with a reciprocal increase in Grade 2 BPD with budesonide. After adjustment for the variables in Table 1, budesonide decreased Severe Type 2 BPD or death (OR 0.48; $0.25-0.92 ; p=0.03$ ) and Grade 3 BPD or death (adjusted OR 0.52; $0.31-0.87 ; p=0.013$ ) compared to the surfactant-only cohort (Supplementary Table S1). 
Table 1. Demographics of cohorts.

\begin{tabular}{llll}
\hline & Surfactant & $\begin{array}{l}\text { Surf }+ \\
\text { budesonide }\end{array}$ & $p$ value \\
\hline Dates of cohorts & $\begin{array}{l}8 / 1 / 2013 \text { to } 7 / \\
\text { 31/2016 }\end{array}$ & $8 / 1 / 16$ to $7 / 31 / 18$ & \\
Infants in cohort & 294 & 173 & \\
Gestational age (mean) & $26.7 \pm 2.1$ & $26.7 \pm 2.1$ & 0.71 \\
Gestational age & $26.4(25.0,28.1)$ & $26.7(25.0,28.4)$ & 0.55 \\
(median) & & & \\
Birth weight (mean) & $846 \pm 205$ & $863 \pm 214$ & 0.39 \\
Birth weight (median) & $825 \mathrm{~g}(674 \mathrm{~g}$, & $850 \mathrm{~g}(680 \mathrm{~g}$, & 0.43 \\
& $1020 \mathrm{~g})$ & $1070 \mathrm{~g})$ & \\
Male & $53 \%(156 / 294)$ & $56 \%(96 / 173)$ & 0.61 \\
Caucasian & $41 \%(123 / 294)$ & $38 \%(65 / 173)$ & 0.38 \\
Outborn infants & $18 \%(53 / 294)$ & $16 \%(27 / 173)$ & 0.52 \\
Antenatal steroids & $83 \%(243 / 294)$ & $80 \%(139 / 173)$ & 0.54 \\
Chorioamnionitis & $57 \%(157 / 274)$ & $51 \%(76 / 150)$ & 0.19 \\
Apgar 5 min & $6.2 \pm 1.9$ & $6.4 \pm 1.8$ & 0.23 \\
CRIB II score (mean) & $10.4 \pm 3.0$ & $10.5 \pm 2.8$ & 0.87 \\
CRIB II score (median) & $10.5(8,13)$ & $10(9,12)$ & 0.95 \\
Surfactant & $1.94 \pm 0.5$ & $2.06 \pm 0.73$ & 0.053 \\
doses (mean) & & & \\
Surfactant doses & $2(2,2)$ & $2(2,2)$ & 0.14 \\
(median) & & & \\
\hline Mean \pm SD; \% (infants & with condition/infants with results & available) \\
Median. (25\%, 75\%) & & & \\
\hline
\end{tabular}

Secondary respiratory outcomes

On the third day of life (DOL 3), infants in the budesonide cohort were less likely to remain intubated ( $43 \%$ vs. $58 \%$; OR 0.55 ; $0.37-0.81 ; p<0.01)$, more likely to be on CPAP ( $43 \%$ vs. $29 \%$; OR $1.9 ; 1.26-2.79 ; p<0.01)$, and had a lower average $\mathrm{FiO}_{2}(0.34 \pm 15$ vs. $30 \pm 11, p=0.008)$. Infants in the budesonide cohort were also less likely to remain intubated on DOL 7 (OR 0.56 ; $0.38-0.82$; $p<0.01)$ and more likely to be on CPAP (OR 1.71; 1.16-2.53; $p<$ 0.01 ), but average $\mathrm{FiO}_{2}$ was similar (Table 2). On DOL 7, the budesonide cohort had a lower percentage of infants with a BPD risk of $>60 \%$ on the NICHD BPD Estimator (OR 0.65; 0.44-0.95; $p=0.03)$. There was also trend toward decreased systemic dexamethasone use in the budesonide cohort $(p=0.07)$. In the subgroups of infants with birth weights $>750 \mathrm{~g}$ or without exposure to chorioamnionitis, budesonide was associated with decreased dexamethasone use (Supplementary Table S2, Supplementary Table S3). Infants exposed to dexamethasone had similar median doses with 13 doses $^{9,24}$ in the surfactant cohort and 10 doses $^{9,24}$ in the budesonide cohort $(p=0.72)$. Infants in the budesonide cohort were again less likely to remain intubated on DOL 28 ( $3 \%$ vs. $10 \%$ OR $0.52 ; 0.31-0.86 ; p=0.015$ ) and more likely to be on CPAP (OR $1.75 ; 1.15-2.59 ; p<0.01$ ). Fewer patients in the budesonide cohort required highfrequency oscillatory ventilation (HFOV) at any point during their NICU stay (OR 0.57; 0.39-0.85; $p<0.01$ ). Respiratory outcomes were similar between the years 2013 and 2015, and changed with the introduction of budesonide and surfactant in 2016 (Supplementary Table S2).

Morbidity outcomes

In the budesonide cohort, the presence of a PDA on any echocardiogram was decreased (OR 0.64; 0.43-0.93; $p=0.03$ ) (Table 3). Additionally, the budesonide cohort received less medical treatment for a PDA (OR 0.52; $0.31-0.81 ; p<0.01)$ and
Table 2. Respiratory outcomes of cohorts.

\begin{tabular}{|c|c|c|c|}
\hline & Surfactant & $\begin{array}{l}\text { Surf }+ \\
\text { budesonide }\end{array}$ & $p$ value \\
\hline \multicolumn{4}{|l|}{ DOL \#7 } \\
\hline IMV/HFOV & $48 \%(135 / 280)$ & $34 \%(57 / 166)$ & $<0.01^{\#}$ \\
\hline NIPPV & $15 \%(42 / 280)$ & $16 \%(26 / 166)$ & 0.89 \\
\hline CPAP & $36 \%(100 / 280)$ & $49 \%(81 / 166)$ & $<0.01^{\#}$ \\
\hline Average $\mathrm{FiO}_{2}$ & $0.31 \pm 0.12$ & $0.30 \pm 0.11$ & 0.12 \\
\hline $\begin{array}{l}\text { NICHD BPD calculator } \\
\text { average }\end{array}$ & $57 \pm 26$ & $52 \pm 27$ & 0.06 \\
\hline $\begin{array}{l}\text { NICHD BPD } \\
\text { calculator } \geq 60 \%\end{array}$ & $55 \%(155 / 280)$ & $45 \%(74 / 166)$ & $0.03^{\#}$ \\
\hline $\begin{array}{l}\text { Postnatal } \\
\text { dexamethasone }\end{array}$ & $51 \%(150 / 294)$ & $42 \%(73 / 173)$ & 0.07 \\
\hline \multicolumn{4}{|c|}{36 weeks corrected gestational age } \\
\hline IMV/NIPPV & $10 \%(26 / 263)$ & $3 \%(5 / 158)$ & $0.01^{\#}$ \\
\hline CPAP & $33 \%(86 / 263)$ & $29 \%(46 / 158)$ & 0.45 \\
\hline $\begin{array}{l}\text { Nasal cannula or } \\
\text { room air }\end{array}$ & $57 \%(151 / 263)$ & $68 \%(107 / 158)$ & $0.04^{\#}$ \\
\hline Death & $11 \%(31 / 294)$ & $9 \%(15 / 173)$ & 0.52 \\
\hline \multicolumn{4}{|l|}{ NICHD BPD definition } \\
\hline No or mild BPD & $29 \%(86 / 294)$ & $31 \%(53 / 173)$ & 0.75 \\
\hline Moderate BPD & $4 \%(12 / 294)$ & $4 \%(7 / 173)$ & 1.0 \\
\hline Severe BPD type I & $47 \%(139 / 294)$ & $54 \%(93 / 173)$ & 0.18 \\
\hline Severe BPD type II & $9 \%(26 / 294)$ & $3 \%(5 / 173)$ & $0.02^{\#}$ \\
\hline $\begin{array}{l}\text { Moderate/severe BPD/ } \\
\text { death }\end{array}$ & $71 \%(208 / 294)$ & $69 \%(120 / 173)$ & 0.75 \\
\hline $\begin{array}{l}\text { Severe Type II BPD } \\
\text { or death }\end{array}$ & $19 \%(57 / 294)$ & $12 \%(20 / 173)$ & $0.03^{\#}$ \\
\hline \multicolumn{4}{|c|}{ NICHD BPD Workshop Grading 2018} \\
\hline No BPD & $29 \%(85 / 294)$ & $31 \%(53 / 173)$ & 0.75 \\
\hline Grade 1 & $14 \%(41 / 294)$ & $9 \%(15 / 173)$ & 0.18 \\
\hline Grade 2 & $26 \%(77 / 294)$ & $40 \%(69 / 173)$ & $<0.01^{\#}$ \\
\hline Grade 3 & $20 \%(31 / 294)$ & $12 \%(21 / 173)$ & $0.02^{\#}$ \\
\hline Grade 3 or death & $31 \%(91 / 294)$ & $21 \%(36 / 173)$ & $0.02^{\#}$ \\
\hline \multicolumn{4}{|c|}{$\begin{array}{l}\text { Mean } \pm \text { SD; } \% \text { (infants with condition/infants with results available) } \\
I M V \text { invasive mechanical ventilation, NIPPV noninvasive positive pressure } \\
\text { ventilation } \\
\# p<0.05 \text { with chi-square test }\end{array}$} \\
\hline
\end{tabular}

underwent surgical ligation less frequently (OR $0.28 ; 0.11-0.75 ; p$ $<0.01)$. No other significant differences were noted between the cohorts for other neonatal morbidities, culture-proven sepsis, or hyperglycemia requiring insulin (Table 3 ).

Growth and discharge outcomes

Infants in the budesonide cohort gained weight at the same rate as infants in the historical cohort; however, they had improved length growth (Table 4). The median GA at discharge was lower in the budesonide cohort ( 39.0 weeks vs. 40.0 weeks, $p<0.01$ ). No infants were back transported to other institutions in the study. There were no significant differences in the gestational ages of the last feed requiring nasogastric tube (NG) assistance or intervention for apnea events to fully explain the shorter length of stay. The average number of risk factors for poor neurodevelopmental outcome (BPD, IVH, ROP ${ }^{32,33}$ ) in the surfactant cohort was $0.9 \pm 0.7$ and the budesonide cohort was $0.8 \pm 0.7(p=0.79)$, and a similar percent of infants had two or more of the three risk factors. 
Subgroup analyses

Birth weight. Similar decreases in ventilator support were found in infants greater or less than $750 \mathrm{~g}$ with budesonide (Supplementary Table S3). Decreases in severe BPD type 2 or death (OR 0.35 ; $0.16-0.76 ; p<0.01$ ), Grade 3 BPD or death (OR 0.38,

Table 3. Neonatal morbidities of cohorts.

\begin{tabular}{|c|c|c|c|}
\hline & Surfactant & $\begin{array}{l}\text { Surf }+ \\
\text { budesonide }\end{array}$ & $p$ value \\
\hline NEC $\geq$ Bell Stage 2 & $7 \%(21 / 294)$ & $6 \%(11 / 173)$ & 0.75 \\
\hline Isolated perforation & $4 \%(12 / 294)$ & $4 \%(7 / 173)$ & 0.99 \\
\hline $\begin{array}{l}\text { Blood culture } \\
\text { positive sepsis }\end{array}$ & $11 \%(32 / 294)$ & $10 \%(17 / 173)$ & 0.84 \\
\hline IVH: Grade III or IV & $16 \%(48 / 294)$ & $16 \%(27 / 173)$ & 0.84 \\
\hline PVL on HUS or MRI & $5 \%(15 / 294)$ & $6 \%(10 / 173)$ & 0.74 \\
\hline VP shunt or reservoir & $4 \%(12 / 294)$ & $5 \%(8 / 173)$ & 0.78 \\
\hline ROP $\geq$ Stage 2 & $18 \%(52 / 294)$ & $15 \%(26 / 173)$ & 0.46 \\
\hline ROP Treatment & $6 \%(18 / 294)$ & $6 \%(11 / 173)$ & 0.87 \\
\hline $\begin{array}{l}\text { PDA on any } \\
\text { echocardiogram }\end{array}$ & $63 \%(185 / 294)$ & $52 \%(90 / 173)$ & $0.02^{\#}$ \\
\hline $\begin{array}{l}\text { Medical treatment } \\
\text { for PDA }\end{array}$ & $35 \%(102 / 294)$ & $22 \%(38 / 173)$ & $<0.01^{\#}$ \\
\hline PDA ligation & $10 \%(28 / 294)$ & $3 \%(5 / 173)$ & $<0.01^{\#}$ \\
\hline Pneumothorax & $7 \%(22 / 294)$ & $6 \%(11 / 173)$ & 0.71 \\
\hline Pulmonary hemorrhage & $15 \%(44 / 294)$ & $9 \%(16 / 173)$ & 0.08 \\
\hline PIE & $18 \%(54 / 294)$ & $21 \%(36 / 173)$ & 0.50 \\
\hline Sildenafil for PHTN & $6 \%(17 / 294)$ & $3 \%(6 / 173)$ & 0.26 \\
\hline Insulin for hyperglycemia & $24 \%(70 / 294)$ & $21 \%(37 / 173)$ & 0.62 \\
\hline \multicolumn{4}{|c|}{$\begin{array}{l}\text { Mean } \pm \text { SD; \% (infants with condition/infants with results available) } \\
N E C \text { necrotizing enterocolitis, } I V H \text { intraventricular hemorrhage, } P V L \\
\text { periventricular leukomalacia, VP ventriculoperitoneal shunt, PDA patent } \\
\text { ductus arteriosus, PIE pulmonary interstitial emphysema } \\
\# p<0.05 \text { with chi-square test }\end{array}$} \\
\hline
\end{tabular}

$0.20-0.73, p<0.01$ ), and the need for HFOV (OR 0.34; 0.18-0.64; $p<0.01$ ) were found in infants $\leq 750 \mathrm{~g}$, whereas infants $>750 \mathrm{~g}$ had decreased exposure to postnatal dexamethasone (OR 0.47; $0.24-0.73 ; p<0.01$ ) and LOS (75 days vs. 83 days; $p=0.02$ ).

Chorioamnionitis. In infants with evidence of histologic chorioamnionitis, there were no significant differences between the two cohorts (Supplementary Table S4) in any respiratory outcomes. In infants that did not have evidence of histologic chorioamnionitis, median LOS was reduced in the budesonide cohort by 17 days ( 77 days vs. 94 days; $p=0.016$ ). There was a reduction in the need for mechanical ventilation in the budesonide cohort at DOL 3 (OR 0.32; $0.17-0.60 ; p<0.01)$ and DOL 7 (OR $0.28 ; 0.14-0.55 ; p<0.01$ ). There was a significant reduction in the use of systemic postnatal dexamethasone in the budesonide cohort (OR 0.40; 0.21-0.74; $p<0.01$ ). In infants born $>$ $750 \mathrm{~g}$ without histologic chorioamnionitis, the budesonide cohort had a decreased rate of moderate or severe BPD or death $(49 \%$ vs. $67 \%$; OR 0.45; 0.22-0.99; $p=0.045$ ).

\section{DISCUSSION}

With the goal of decreasing our BPD rates, we began administering a combination of budesonide and surfactant to all infants with a birth weight $\leq 1250 \mathrm{~g}$ who failed CPAP or required delivery room intubation. To ensure safety of our clinical practice change, we collected data on respiratory and neonatal outcomes quarterly for the first 2 years of budesonide use. Unlike the studies by Yeh et al., which necessitated high oxygen requirements, we gave the combination to any infant who otherwise would have qualified for surfactant therapy. The budesonide was mixed with surfactant at the bedside at our level IV NICU, our delivery hospital, or by our transport team at outside hospitals, and additional doses were given based on clinical parameters at $6-8 \mathrm{~h}$ after the first dose. We found many short-term respiratory benefits between the two cohorts, but the overall BPD rate did not decrease. We did not find any short-term morbidities (hyperglycemia requiring insulin or sepsis) or increases in adverse secondary neonatal outcomes with the addition of budesonide to surfactant. We did not collect

Table 4. Length of stay, discharge statistics.

\begin{tabular}{|c|c|c|c|}
\hline Median LOS & 94 (75-118) days & 89 (66.5-113) days & 0.06 \\
\hline Median GA at discharge & $40.0(38.1-43.0) \mathrm{wk}$ & $39.0(37.2-41.9) \mathrm{wk}$ & $<0.01^{\mathrm{t}}$ \\
\hline Death & $10.5 \%(31 / 294)$ & $8.7 \%(15 / 173)$ & 0.53 \\
\hline Nasal cannula at discharge & $46 \%(122 / 263)$ & $47 \%(75 / 158)$ & 0.84 \\
\hline Tracheostomy placement & $3.0 \%(8 / 263)$ & $2.5 \%(4 / 158)$ & 0.99 \\
\hline GA at last NG feed (median) & $37.6(35.9,39.7) \mathrm{wk}$ & $37.1(35.6,38.9) \mathrm{wk}$ & 0.29 \\
\hline GA at last apnea (mean) & $37.3 \pm 4.4 w k$ & $37.6 \pm 4.7 w k$ & 0.61 \\
\hline GA at last apnea (median) & $37.0(35.1,39.0) \mathrm{wk}$ & $36.9(35.1,39.0) \mathrm{wk}$ & 0.77 \\
\hline GA apnea or NG (mean) & $39.5 \pm 4.6 \mathrm{wk}$ & $39.0 \pm 4.9 w k$ & 0.41 \\
\hline GA apnea or NG (median) & $38.6(36.8,41.0) \mathrm{wk}$ & $37.9(36.1,40.6) \mathrm{wk}$ & 0.052 \\
\hline Two or more risk factors for poor neurodevelopment or death & $23.5 \%(69 / 294)$ & $22.0 \%(38 / 173)$ & 0.79 \\
\hline
\end{tabular}


neurodevelopmental outcomes so are unable to discuss longer term morbidities.

Similar to the previous clinical trials by Yeh et al. and with early postnatal steroids, we found an association with decreased requirements for mechanical ventilation. ${ }^{4}$ This is likely due to the anti-inflammatory effects of budesonide or a direct maturational effect on the preterm lung. ${ }^{16}$ The infants who received budesonide tended to have less postnatal steroid exposure ( $p=$ 0.07), with decreases in exposure of $40 \%$ in infants with birth weights $>750 \mathrm{~g}$ and infants without histologic chorioamnionitis. In infants $\leq 750 \mathrm{~g}$, decreases in invasive respiratory support were found, but most of these infants $(73 \%)$ still had a risk of BPD calculated at $>60 \%$ and received postnatal steroids. Although decreasing respiratory support early in hospitalization should affect BPD, we did not find a sustained benefit with early budesonide with surfactant.

Our overall BPD or death rates were higher $(71 \%$ in surfactant and $69 \%$ in budesonide cohort) than in other studies and higher than predicted by the NICHD BPD calculator on DOL 7. In a metaanalysis of the two studies by Yeh et al., the rate of BPD or death decreased by $20 \%$, with an odds ratio of $0.34[0.23,0.53].]^{7,12,34}$ Low-dose hydrocortisone also reduced BPD or death from 49 to $40 \%$ in a similar patient population. ${ }^{3}$ In the Bassler et al. study of inhaled budesonide, the BPD or death rate also decreased from 46 to $40 \%$, but there was an increased mortality rate with inhaled budesonide. ${ }^{5,18}$ The rate of death in our cohorts ( $8.7 \%$ vs. $\left.10.5 \%\right)$ was not significantly different. Other studies with inhaled budesonide have not demonstrated an increased risk of mortality, and the average dose of budesonide in our cohort $(0.44 \mathrm{mg})$ was considerably smaller than the average total dose $(18,800 \mu \mathrm{g}$ or $18.8 \mathrm{mg}$ ) in the inhaled budesonide trial. ${ }^{11,35}$ Although the overall $B P D$ rate did not change, the severity of BPD decreased, with less infants requiring mechanical ventilation at 36 weeks CGA (severe BPD type 2) and more infants on room air or nasal cannula. The rates of Grade 3 BPD (IMV or NIPPV/CPAP/HFNC with $\mathrm{FiO}_{2}>0.30$ ) were also lower in the budesonide exposed infants. ${ }^{28}$

Since budesonide has about a 200-fold increased affinity for the glucocorticoid receptor relative to cortisol and can be released from the lung, it is important to monitor other organ systems. ${ }^{16,36,37}$ Most of the secondary outcomes were similar between the two cohorts (Table 3). The presence of a PDA on any echocardiogram was lower in the budesonide cohort, which was also observed by Yeh et al. and with early postnatal steroids., ${ }^{4,35}$ Treatment of a PDA with medication or ligation also decreased, but this could be due to changes in clinical practice over time. One of the most interesting secondary outcomes were the differences in LOS and GA at discharge. The median LOS in the surfactant-only cohort was comparable to control babies (93 days) in the inhaled budesonide study. ${ }^{35}$ The decreased age at discharge in the budesonide cohort was not explained by improved oral feeding or last apnea requiring intervention, though the GA when both criteria were met was nearly 5 days earlier $(p=0.07)$. Although weight gain per day was similar, infants in the budesonide cohort had a larger increase in length per day.

The two largest factors effecting the response to budesonide were birth weight and histologic chorioamnionitis. In subgroup analysis, the presence of chorioamnionitis negated the association of beneficial effects with budesonide. In the setting of mechanical ventilation, chorioamnionitis increases the risk of BPD. ${ }^{38}$ The rates of histologic chorioamnionitis were much lower $(10-20 \%)$ in the previous studies of intratracheal and inhaled budesonide, and may explain the decreased effect on BPD. ${ }^{7,35}$ Although benefits on respiratory support were found in all birth weight groups, budesonide had the largest effect on infants born $>750 \mathrm{~g}$, with an additional association of a shortened median LOS by 8 days and decreased postnatal steroids. Infants born $\leq 750 \mathrm{~g}$ received more surfactant (2.3 vs. 2.1 doses) in the budesonide cohort, whereas surfactant dosing was similar between other subgroups.
In infants born $>750 \mathrm{~g}$ without chorioamnionitis, budesonide was associated with a decrease in moderate or severe BPD or death by $18 \%(p<0.05)$, which is similar to studies with low chorioamnionitis rates.

There are limitations inherent to cohort studies, as they are hypothesis generating and causality cannot be proven. Clinical practice could have changed over the time of the study and there may be factors unaccounted for in a cohort comparison that could affect outcomes. To this end, many of the protocols governing clinical care within our practice existed before both cohorts (August 2013 to August 2018). Our unit began using bubble CPAP respiratory support with a RAM cannula in the delivery room and NICU around 2011 and have used tidal volume ventilation as our primary mode of ventilation throughout study. ${ }^{22}$ The NICU began a feeding protocol in 2012 and donor breast milk use in 2010. Our postnatal dexamethasone protocol, based on the NICHD BPD calculator, started in the beginning of 2013 and BPD risk scores appear in patient problem lists. The BPD calculator is a risk estimator and not typically used as an outcome itself. It was included to more subjectively analyze the postnatal dexamethasone use. Respiratory information was extracted electronically from EPIC, and a 36-week CGA room air challenge was not always performed; thus the BPD rate could be lower in both groups. An analysis of respiratory outcomes based on year within the cohorts (Supplementary Table S2) demonstrates a stable rate of outcomes until the introduction of budesonide. The inclusion of the severity of BPD is only recently being used in the neonatal literature; thus the combined outcome of BPD or death is the primary outcome. Pathologic reports were only available on $88 \%$ of infants so some infants could not be classified based on chorioamnionitis and this could affect the subgroup analysis. The largest limitation is the lack of long-term follow-up data on the infants in the cohorts. Previous studies of inhaled budesonide or budesonide with surfactant did not find changes in neurodevelopment in survivors. ${ }^{5,7}$ Using the predictive model for poor neurodevelopment by Schmidt et al., validated in two randomized trials, ${ }^{32,33}$ we find no differences in the number of risk factors for poor neurodevelopment between groups (Table 4).

\section{CONCLUSIONS}

In this observational cohort comparison, the addition of budesonide to surfactant for the treatment of RDS was associated with a decrease in the severity of BPD without changing the overall incidence of BPD. Infants in the budesonide cohort were extubated earlier, required less use of HFOV, and met criteria for postnatal dexamethasone less often. The introduction of budesonide into clinical practice was not difficult, but the effects on BPD were not as dramatic as found in the previous randomized clinical trials. The largest effects associated with the introduction of budesonide were found in infants with a birth weight $>750 \mathrm{~g}$ and no chorioamnionitis. The overall rates of BPD and histologic chorioamnionitis at an individual institution could influence the effectiveness of the intervention. Additional large randomized trials using similar criteria to our cohorts should be conducted on this promising treatment combination.

\section{ACKNOWLEDGEMENTS}

This study was supported by the Department of Pediatrics and SSM Cardinal Glennon Foundation.

\section{AUTHOR CONTRIBUTIONS}

N.H.H. and T.B.K. conceptualized and designed the study, extracted the data, analyzed the data, drafted the initial manuscript, and reviewed and revised the manuscript. F.H.S., C.A. and N.B. conceptualized and designed the study, extracted 
the data, and reviewed and revised the manuscript. H.L.W. designed the study, extracted the data, and reviewed and revised the manuscript. All authors approved the final manuscript as submitted.

\section{ADDITIONAL INFORMATION}

The online version of this article (https://doi.org/10.1038/s41390-019-0663-6) contains supplementary material, which is available to authorized users.

Competing interests: The authors declare no competing interests.

Publisher's note Springer Nature remains neutral with regard to jurisdictional claims in published maps and institutional affiliations.

\section{REFERENCES}

1. Laughon, M. M. et al. Prediction of bronchopulmonary dysplasia by postnatal age in extremely premature infants. Am. J. Respir. Crit. Care Med. 183, 1715-1722 (2011).

2. Ambalavanan, N. et al. Cytokines associated with bronchopulmonary dysplasia or death in extremely low birth weight infants. Pediatrics 123 1132-1141 (2009).

3. Baud, O. et al. Effect of early low-dose hydrocortisone on survival without bronchopulmonary dysplasia in extremely preterm infants (PREMILOC): a doubleblind, placebo-controlled, multicentre, randomised trial. Lancet 387, 1827-1836 (2016).

4. Doyle, L. W., Cheong, J. L., Ehrenkranz, R. A. \& Halliday, H. L. Early ( $<8$ days) systemic postnatal corticosteroids for prevention of bronchopulmonary dysplasia in preterm infants. Cochrane Database Syst. Rev. 10, CD001146 (2017)

5. Bassler, D. et al. Long-term effects of inhaled budesonide for bronchopulmonary dysplasia. N. Engl. J. Med. 378, 148-157 (2018)

6. Huang, L. T., Yeh, T. F., Kuo, Y. L., Chen, P. C. \& Chen, C. M. Effect of surfactant and budesonide on the pulmonary distribution of fluorescent dye in mice. Pediatrics Neonatol. 56, 19-24 (2015).

7. Yeh, T. F. et al. Intratracheal administration of budesonide/surfactant to prevent bronchopulmonary dysplasia. Am. J. Respir. Crit. Care Med. 193, 86-95 (2016).

8. van den Brink, K. I. et al. Evidence of the in vivo esterification of budesonide in human airways. Br. J. Clin. Pharmacol. 66, 27-35 (2008).

9. Brattsand, R. \& Miller-Larsson, A. The role of intracellular esterification in budesonide once-daily dosing and airway selectivity. Clin. Therapeut. 25(Suppl C) C28-C41 (2003).

10. Bassler, D. et al. Early inhaled budesonide for the prevention of bronchopulmonary dysplasia. N. Engl. J. Med. 373, 1497-1506 (2015).

11. Shinwell, E. S., Portnov, I., Meerpohl, J. J., Karen, T. \& Bassler, D. Inhaled corticosteroids for bronchopulmonary dysplasia: a meta-analysis. Pediatrics 138 e20162511 (2016).

12. Yeh, T. F. et al. Early intratracheal instillation of budesonide using surfactant as a vehicle to prevent chronic lung disease in preterm infants: a pilot study. Pediatrics 121, e1310-e1318 (2008)

13. Venkataraman, R., Kamaluddeen, M., Hasan, S. U., Robertson, H. L. \& Lodha, A Intratracheal administration of budesonide-surfactant in prevention of bronchopulmonary dysplasia in very low birth weight infants: a systematic review and meta-analysis. Pediatr. Pulmonol. 52, 968-975 (2017).

14. Ricci, F. et al. In vitro and in vivo characterization of poractant alfa supplemented with budesonide for safe and effective intratracheal administration. Pediatr. Res. 82, 1056-1063 (2017).

15. Li, L. et al. Effects of intratracheal budesonide during early postnatal life on lung maturity of premature fetal rabbits. Pediatr. Pulmonol. 53, 28-35 (2018).
16. Kothe, T. B. et al. Effects of budesonide and surfactant in preterm fetal sheep. Am J. Physiol. Lung Cell Mol. Physiol. 315, L193-L201 (2018).

17. Kothe, T. B. et al. Surfactant plus budesonide decreases lung and systemic inflammation in mechanically ventilated preterm sheep. Am. J. Physiol. Lung Cell Mol. Physiol. 316, L888-L893 (2019).

18. Barrette, A. M. et al. Antiinflammatory effects of budesonide in human fetal Lung Am. J. Respir. Cell Mol. Biol. 55, 623-632 (2016).

19. Moore, C. D. et al. Metabolic pathways of inhaled glucocorticoids by the CYP3A enzymes. Drug Metab. Dispos. Biol. Fate Chem. 41, 379-389 (2013).

20. O'Connell, E. J. Review of the unique properties of budesonide. Clin. Therapeut. 25 (Suppl C), C42-C60 (2003).

21. Roberts, J. K. et al. Pharmacokinetics of budesonide administered with surfactant in premature lambs: implications for neonatal clinical trials. Curr. Clin. Pharm. 11 53-61 (2016)

22. Claassen, C. C., Hillman, N. H., Brown, K., Williams, H. L. \& Strand, M. L. Comparison of bubble CPAP devices using RAM cannula for extubation failure in very low birth weight infants: randomized and cohort studies. Neonatology 115, 28-35 (2019).

23. Finer, N. N. et al. Early CPAP versus surfactant in extremely preterm infants. $N$. Engl. J. Med 362, 1970-1979 (2010)

24. Parry, G., Tucker, J. \& Tarnow-Mordi, W., Group UKNSSC. CRIB II: an update of the clinical risk index for babies score. Lancet 361, 1789-1791 (2003).

25. Ehrenkranz, R. A. et al. Validation of the National Institutes of Health consensus definition of bronchopulmonary dysplasia. Pediatrics 116, 1353-1360 (2005).

26. Jobe, A. H. \& Bancalari, E. Bronchopulmonary dysplasia. Am. J. Respir. Crit. Care Med. 163, 1723-1729 (2001).

27. Abman, S. H. et al. Interdisciplinary care of children with severe bronchopulmonary dysplasia. J. Pediatr. 181, 12-28 e1 (2017).

28. Higgins, R. D. et al. Bronchopulmonary dysplasia: executive summary of a workshop. J. Pediatr. 197, 300-308 (2018)

29. Doyle, L. W. et al. Low-dose dexamethasone facilitates extubation among chronically ventilator-dependent infants: a multicenter, international, randomized, controlled trial. Pediatrics 117, 75-83 (2006).

30. Juhl, S. M., Hansen, M. L., Gormsen, M., Skov, T. \& Greisen, G. Staging of necrotising enterocolitis by Bell's criteria is supported by a statistical pattern analysis of clinical and radiological variables. Acta Paediatr. 108, 842-848 (2019).

31. Quinn, G. E. et al. Incidence and early course of retinopathy of prematurity: secondary analysis of the postnatal growth and retinopathy of prematurity (G ROP) study. JAMA Ophthalmol. 136, 1383-1389 (2018).

32. Schmidt, B. et al. Impact of bronchopulmonary dysplasia, brain injury, and severe retinopathy on the outcome of extremely low-birth-weight infants at 18 months: results from the trial of indomethacin prophylaxis in preterms. JAMA 289, 1124-1129 (2003).

33. Schmidt, B. et al. Prediction of late death or disability at age 5 years using a count of 3 neonatal morbidities in very low birth weight infants. J. Pediatr. 167, 982-986 e2 (2015).

34. Boel, L., Banerjee, S. \& Chakraborty, M. Postnatal steroids in extreme preterm infants: Intra-tracheal instillation using surfactant as a vehicle. Paediatr. Respir. Rev. 25, 78-84 (2018).

35. Bassler, D. et al. Early inhaled budesonide for the prevention of bronchopulmonary dysplasia. N. Engl. J. Med. 373, 1497-1506 (2015).

36. Esmailpour, N., Hogger, P. \& Rohdewald, P. Binding kinetics of budesonide to the human glucocorticoid receptor. Eur. J. Pharm. Sci. 6, 219-223 (1998).

37. Kothe, T. B. R. E. et al. Surfactant plus budesonide decreases lung and systemic inflammation in mechanically ventilated preterm sheep. AJP-Lung $\mathbf{3 1 6}$ L888-L893 (2019).

38. Van Marter, L. J. et al. Chorioamnionitis, mechanical ventilation, and postnatal sepsis as modulators of chronic lung disease in preterm infants. J. Pediatr. 140, 171-176 (2002). 